\title{
Vertical motion of particles in vibration-induced granular capillarity
}

\author{
Fengxian Fan $^{1, *}, J u$ Liu $^{1}$, Eric $J R$ Parteli $^{2}$ and Thorsten Pöschel $^{3}$ \\ ${ }^{1}$ Shanghai Key Laboratory of Multiphase Flow and Heat Transfer in Power Engineering, University of Shanghai for Science and \\ Technology, 200093 Shanghai, China \\ ${ }^{2}$ Department of Geosciences, University of Cologne, 50969 Cologne, Germany \\ ${ }^{3}$ Institute for Multiscale Simulation, Universität Erlangen-Nürnberg, D-91052 Erlangen, Germany
}

\begin{abstract}
When a narrow tube inserted into a static container filled with particles is subjected to vertical vibration, the particles rise in the tube, much resembling the ascending motion of a liquid column in a capillary tube. To gain insights on the particle dynamics dictating this phenomenon - which we term granular capillarity - we numerically investigate the system using the Discrete Element Method (DEM). We reproduce the dynamical process of the granular capillarity and analyze the vertical motion of the individual particles in the tube, as well as the average vertical velocities of the particles. Our simulations show that the height of the granular column fluctuates in a periodic or period-doubling manner as the tube vibrates, until a steady-state (capillary) height is reached. Moreover, our results for the average vertical velocity of the particles in the tube at different radial positions suggest that granular convection is one major factor underlying the particle-based dynamics that lead to the granular capillarity phenomenon.
\end{abstract}

\section{Introduction}

When vertical vibration is introduced into granular systems, the granular materials can exhibit a diversity of interesting phenomena, such as size segregation, density waves, convection and heap formation [1]. A particularly interesting phenomenon is that when a narrow tube inserted into a static container filled with particles is subjected to vertical vibration, the particles rise in the tube. Because this process resembles the rise of a liquid in a capillary tube, we term it granular capillarity. This phenomenon has attracted the interest of many researchers since it offers a potential means to pump or lift granular systems [2].

Since the first experimental observations of granular capillarity by Liu et al. [2], much effort has been made to unravel and model its underlying mechanisms [3-5]. In particular, particle-based numerical models have been developed to simulate the particle dynamics due to the vibrating tube [4]. However, these simulations were performed for one oscillation cycle of the vertically vibrating tube only, and thus they could not reproduce the rising process of the granular column in the tube observed experimentally in the course of many oscillation cycles. Moreover, it remains uncertain which physical mechanisms dictate the ascending motion of the granular column in the tube [5].

Numerical simulations using the Discrete Element Method (DEM) [6] can offer a helpful tool in the investigation of the granular capillarity phenomenon. In the DEM, Newton's equations of translational and rotational motion for all particles are simultaneously solved by taking into account the forces and torques acting on each particle. Here we use three-dimensional (3D) DEM simulations to reproduce the experimentally observed granular capillarity phenomenon and investigate the particle-based processes that lead to referred phenomenon. We will show that the granular capillarity effect is associated with granular convection in the system, which is inherent to particulate materials subject to vibrations.

\section{DEM model}

According to the DEM, the equations for the translational and rotational motion of particle $i$ with radius $R_{i}$, mass $m_{i}$ and moment of inertia $I_{i}$, read

$$
\begin{gathered}
m_{i} \frac{\mathrm{d} \mathbf{v}_{i}}{\mathrm{~d} t}=\sum_{j=1}^{n}\left(\mathbf{F}_{i j}^{n}+\mathbf{F}_{i j}^{t}\right)+m_{i} \mathbf{g} \\
I_{i} \frac{\mathrm{d} \omega_{i}}{\mathrm{~d} t}=\sum_{j=1}^{n}\left(\mathbf{M}_{i j, t}+\mathbf{M}_{i j, r}\right)
\end{gathered}
$$

where $\mathbf{v}_{i}$ and $\omega_{i}$ are the translational and angular velocities of particle $i, t$ is the time, $n$ is the number of particles in contact with particle $i, \mathbf{g}$ is the acceleration of gravity, while $\mathbf{F}_{i j}^{n}$ and $\mathbf{F}_{i j}^{t}$ are the interaction forces between particle $i$ and particle $j$ in normal and tangential directions, respectively. Moreover, the torques acting on particle $i$ are $\mathbf{M}_{i j, t}$ due to the tangential force $\mathbf{F}_{i j}^{t}$, and $\mathbf{M}_{i j, r}$ due to the rolling resistance.

* Corresponding author: fanfengxian@ $@$ hotmail.com 
We assume viscoelastic interaction [8] for the normal force $\mathbf{F}_{i j}^{n}$ and apply a modified Cundall-Stract model [6] for the tangential force $\mathbf{F}_{i j}^{t}$. Hence the forces read

$$
\begin{gathered}
\mathbf{F}_{i j}^{n}=\min \left(0,-\rho \delta_{n i j}^{3 / 2}-\frac{3}{2} A_{n} \rho \sqrt{\delta_{n i j}} \dot{\delta}_{n i j}\right) \mathbf{e}_{n} \\
\mathbf{F}_{i j}^{t}=-\min \left(\mu_{s}\left|\mathbf{F}_{i j}^{n}\right|, \int_{\text {path }} \frac{4 G}{2-v} \sqrt{R_{\text {eff }} \delta_{n i j}} \mathrm{~d} s+\right. \\
\left.A_{t} \sqrt{R_{\text {eff }} \delta_{n i j}} v_{t i j}\right) \mathbf{e}_{t}
\end{gathered}
$$

where $\delta_{n i j}=R_{i}+R_{j}-\left|\mathbf{r}_{i}-\mathbf{r}_{j}\right|$ is the overlap distance between the particles at positions $\mathbf{r}_{i}$ and $\mathbf{r}_{j}, \mathbf{e}_{n}=$ $\left(\mathbf{r}_{j}-\mathbf{r}_{i}\right) /\left(\left|\mathbf{r}_{j}-\mathbf{r}_{i}\right|\right)$ is the normal unit vector, $\rho$ is the elastic parameter, which is a function of the Young's modulus, $Y$, the Poisson's ratio, $v$, and the effective radius, $R_{\mathrm{eff}}=R_{i} R_{j} /\left(R_{i}+R_{\mathrm{j}}\right)$,

$$
\rho=\frac{2 Y}{3\left(1-v^{2}\right)} \sqrt{R_{\text {eff }}}
$$

and $A_{n}$ is the dissipative parameter, which depends not only on the Young's modulus and the Poisson's ratio, which are easily available for a variety of materials, but also on the material viscosities, which are not directly available. In order to determine $A_{n}$, we use an expression of the coefficient of restitution, $\varepsilon$, for the collision of the particles $i$ and $j$ [9]. Moreover, $\mu_{s}$ is the sliding friction coefficient, $G=Y /[2(1+v)]$ is the shear modus, $v_{t i j}$ is the relative tangential velocity at the point of contact, $A_{i}=A_{n} Y /\left(1-v^{2}\right)$ is the tangential dissipative parameter [10] and $\mathbf{e}_{t}$ is the tangential unit vector. The integral in Eq. (4) is performed over the displacement of the particles at the point of contact for the duration of the contact.

According to the directional constant torque model [11], the torque associated with the rolling resistance, $\mathbf{M}_{i j, r}$, can be expressed as

$$
\mathbf{M}_{i j, r}=-\mu_{r} \rho \delta_{n i j}{ }^{3 / 2} \frac{\omega_{i j}}{\left|\omega_{i j}\right|} R_{\text {eff }}
$$

where $\mu_{r}$ is the coefficient of rolling resistance and $\omega_{i j}$ is the relative angular velocity between particle $i$ and particle $j$.

In the DEM simulation, the time step $\Delta t$ must be small enough to make sure that the Newton's equations are accurately solved. In this work, we take $\Delta t=t_{\mathrm{col}} / 30$, where $t_{\text {col }}$ is the collision time, which can be estimated using the collision time for the center-to-center collision of frictionless elastic particles

$$
t_{\mathrm{col}} \approx 3.21\left(\frac{m_{\mathrm{eff}}}{\rho}\right)^{2 / 5} v_{\mathrm{imp}}^{-1 / 5}
$$

where $m_{\mathrm{eff}}=m_{i} m_{j} /\left(m_{i}+m_{j}\right)$ is effective mass, and $v_{\mathrm{imp}}$ is the impact velocity. In this work, a reference impact velocity $v_{\text {imp }}=1.0 \mathrm{~m} / \mathrm{s}$ is adopted to compute $t_{\text {col }}$.

\section{Numerical experiments}

Our simulation system consists of $N=200,000$ monosized particles with diameter $d_{p}=0.6 \mathrm{~mm}$ and density $\rho_{p}=2500 \mathrm{~kg} / \mathrm{m}^{3}$. The particles are filled in a cylindrical silo with inner diameter $D=28.2 \mathrm{~mm}$, while the tube has inner diameter $d=8 \mathrm{~mm}$ and thickness $\delta=0.6 \mathrm{~mm}$. The tube is inserted into the center of the silo and filled up to the same level as the granular surface in the other volume. The lower end of the tube is initially at $z=0 \mathrm{~mm}$, and the bottom of the silo is at $z=-30 \mathrm{~mm}$, while the heights of the silo and the tube are large enough to contain all the particles during the simulation. The frictional wall boundary condition is applied for the walls of both silo and tube. The equations used for computing the forces between particles and the walls are the same used for modelling particle-particle collisions where one of the contact partners is of infinite mass and radius.

We vibrate the tube by applying a vertical displacement $z$ which is a sinusoidal function of time,

$$
z=A \sin (2 \pi f t)
$$

where $A$ and $f$ are the amplitude and the frequency, respectively. In this work, $A=12.42 \mathrm{~mm}$ and $f=10 \mathrm{~Hz}$ are applied. The physical properties of the particles used in the simulation are listed in Table 1. The simulations were performed with 4 processors in the workstation cluster equipped with Intel Core i7-4790 CPUs. It took about 45 hours to run a simulation case for the tube vibration time of $100 T$ ( $T$ is the period of tube vibration).

Table 1. Physical properties of the particles.

\begin{tabular}{lcc}
\hline \multicolumn{1}{c}{ Parameter } & Symbol & Value \\
\hline Young's modulus & $Y$ & $10 \mathrm{MPa}$ \\
Poisson's ratio & $v$ & 0.25 \\
Coefficient of restitution & $\varepsilon$ & 0.5 \\
Particle-particle sliding friction coefficient & $\mu_{s, p p}$ & 0.8 \\
Particle-wall sliding friction coefficient & $\mu_{s, p w}$ & 0.58 \\
Particle-particle rolling friction coefficient & $\mu_{r, p p}$ & 0.1 \\
Particle-wall rolling friction coefficient & $\mu_{r, p w}$ & 0.05 \\
\hline
\end{tabular}

\section{Results and discussion}

\subsection{Phenomenon of granular capillarity}

Fig. 1 shows snapshots of the simulation of the granular capillarity phenomenon. The snapshots show twodimensional cuts of the granular system along the tube axis, the color of each particle indicates its initial position at $t=0$. Particles which, at $t=0$, are within the cylindrical shell centered at the tube's central axis with radius equal to the tube's radius, are colored blue, while particles outside this shell at $t=0$ are colored red. The particles keep this color code throughout the simulation, independently of how individual particle positions change with time. The simulation starts with the free surface of the particles in the tube and in the silo levelled. Due to the friction between the particles and the tube wall, the particles in the tube rise once the tube moves upwards, leaving a void on the bottom of the tube. This void is filled rapidly by the nearby particles in the silo, which enter the tube through its bottom end thereby increasing the height of the granular column in the tube. That is, the aforementioned void filling mechanism leads 
to an increase of the granular column height in the first oscillation cycles of the tube.

However, after several oscillation cycles $(\sim 70 T), H_{\mathrm{c}}$ slowly achieves a steady-state value, as can be seen from Fig. 2 where we plot the granular column height as a function of time. We see from this figure that $H_{\mathrm{c}}$ fluctuates as the tube vibrates. The period and amplitude of the fluctuation change with the vibration time. It should be noted that during $t=0 \sim 2 T$, the large fluctuation of $H_{\mathrm{c}}$ is caused by inertial effects. After the initial stage, the evolution of $H_{\mathrm{c}}$ shows three stages in the granular capillarity: the periodic rising stage, the period-doubling rising stage and the period-doubling terminal stage. In the periodic rising stage, $H_{\mathrm{c}}$ fluctuates with the same period as the tube vibration as shown during $2 T<t<9 T$. When $t>9 T$, the development of the granular capillarity turns to a period-doubling stage, where $H_{\mathrm{c}}$ oscillates in a period of $2 T$ and with a much larger amplitude compared with the periodic stage. As the time increases further to $70 T, H_{\mathrm{c}}$ approaches a steady-state value. It has been observed that the appearance and duration of the above stages differs greatly with the change of the vibration condition and the sizes of the tube and the silo.

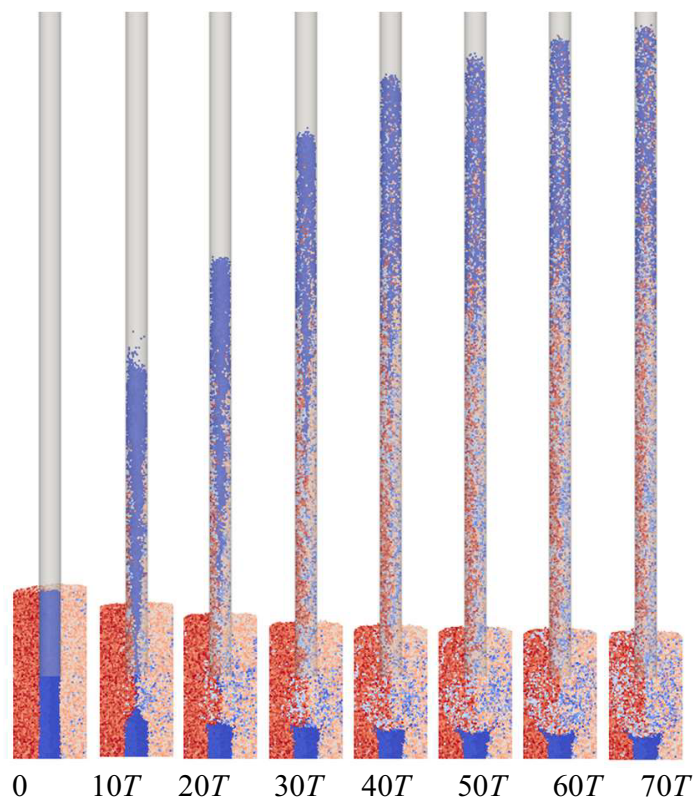

Fig. 1. Dynamic process of granular capillarity.

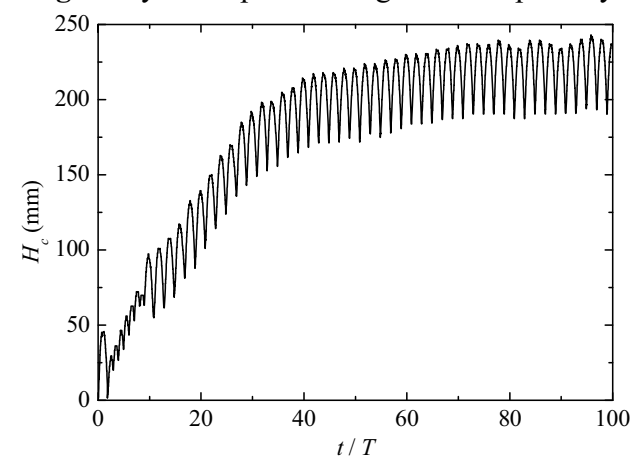

Fig. 2. Height of the granular column as a function of time.

\subsection{Vertical motion of individual particles}

We show the vertical displacements and velocities of three individual particles at different vertical positions along the tube in Fig. 3. It can be seen from Fig. 3a that, in the considered range $5<t / T<15$, all three particles a$c$ rise along the tube but their ascending motion is modulated by oscillations of different magnitudes. Indeed, we see that the higher the vertical position the larger the amplitude. We have found that the uppermost layers of the granular column have a smaller solid fraction compared to the center and bottom of the tube, which explains why the modulation amplitude for particle "c" is larger than for particles " $\mathrm{b}$ " and "a". However, we are currently investigating the particlebased processes underlying the rise of the granular column in more detail and the referred behavior still remains to be elucidated. Moreover, we note that there is a phase lag between the oscillation of the tube and the vertical particle trajectories for all particles. It is still uncertain how this phase lag is related to the transition of the periodic rising stage to the period-doubling rising stage mentioned in the previous section. However, it is observed that in the period-doubling stage, the particle in the top oscillates with a period of $2 T$, whereas the particles in the bottom undergoes a transition from periodic to period-doubling oscillation.

Generally, once the tube starts to move upward from its neutral position with its bottom end at $z=0$, the particles experience decelerated rising, accelerated falling, decelerated falling, and accelerated rising in a cycle of tube vibration, as shown in Fig. 3(b). The downward acceleration during the particles' decelerated rising and accelerated falling is approximately $g$ on average, indicating that the gravity is the dominant force. On the other hand, the average upward acceleration during the particles' decelerated falling and accelerated rising is approximately $7 g$, which is larger than the maximum acceleration of the tube vibration $\left(A \omega^{2} \approx 5 g\right)$. It indicates that the upward acceleration is caused not only by the friction but also by the collision between the particles.
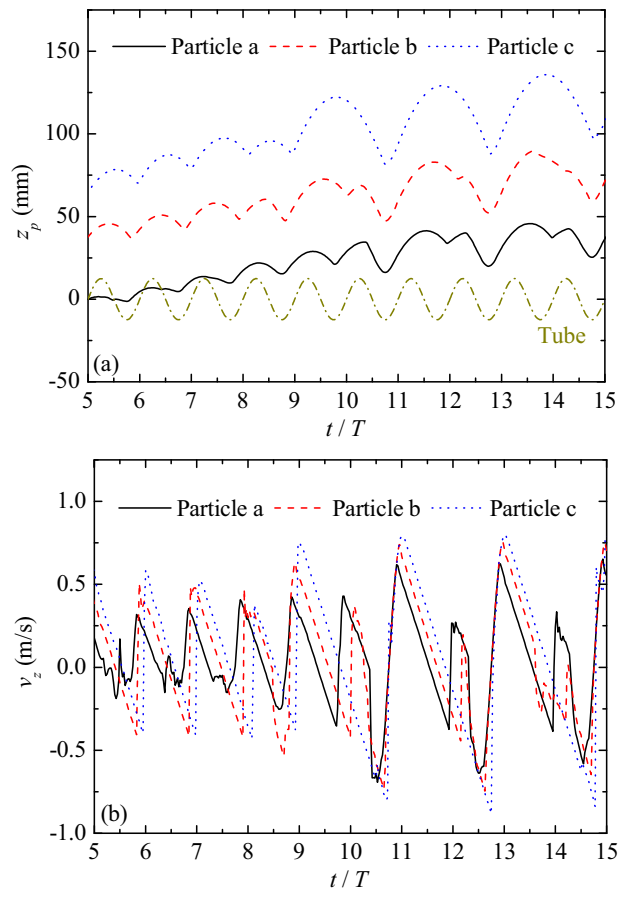

Fig. 3. Vertical motion of individual particles. 


\subsection{Average vertical velocity of particles}

The average vertical velocity of the particles in the tube during different stages of the granular capillarity as a function of dimensionless radial position is presented in Fig. 4. In the periodic rising stage, the rising velocity of the particles near the wall is larger than in the center, while the rising velocity of the particles in the center is larger than near the wall in the period-doubling stage. Moreover, the rising velocity is smaller in the perioddoubling rising stage compared with the periodic rising stage. During the periodic doubling steady-state stage, the particles in the tube center move upward whereas the particles near the tube wall move downward, indicating the occurrence of granular convection. Due to the convective motion of the particles in the tube, the net particle mass fluxes near the walls and in the center compensate each other, and thus the average height of the granular column achieves a stable value.

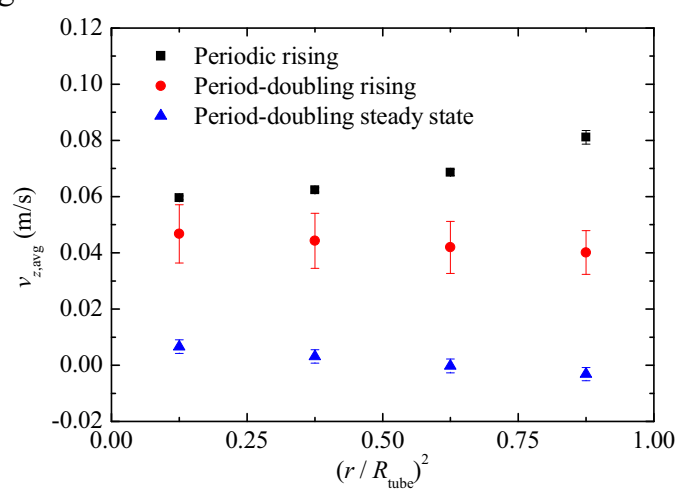

Fig. 4. Average vertical velocity of the particles in the tube as a function of dimensionless radial position $\left(r / R_{\text {tube }}\right)^{2}$.

Therefore, our simulations show that the phenomenon of granular capillarity is related to the process of granular convection which is characteristic of granular systems subject to vibrations. We have seen that, as the tube vibrates, convection occurs within the granular material in the tube's outer volume (that is, in the silo). Indeed, we have performed simulations without frictional forces between particles and the silo's walls and found that, under this condition, the granular column does not rise along the tube. Moreover, we also found that simulations using periodic boundary conditions in the lateral directions also showed no granular capillary effect. These observations lead us to the conclusion that the interactions between the particles and the frictional walls of the silo play an important role for the capillary behavior of the granular column in the tube [12]. However, future work is needed to characterize the convection mechanism in the silo and the tube, as well as to explain whether and how this mechanism affects the capillary phenomenon.

\section{Conclusions}

In conclusion, we have presented the simulation of the experimentally reported granular capillarity phenomenon [2-5,14], which was caused by vertical vibrations of a vertical tube inserted in a container filled with particles. It is interesting to note that, in previous experiments, vertical vibrations were applied to the silo instead of the tube, and the phenomenon also occurred. Therefore, our simulations suggest that the phenomenon is robust upon whether the silo or the tube is subject to the vibrations.

It is worthy noting that the granular matter exhibits rich dynamic behavior under vertical vibration, depending on the system size, the vibration condition and the amount of particles. Future work is thus necessary to examine the effects of the parameters mentioned above. Moreover, it is still uncertain how the phenomenon of jamming, inherent to granular flows through pipes and narrow channels, may be influencing particle behavior and the capillary height. To clarify this role, more systematic investigation under different conditions of solid fraction, frictional properties and texture of the tube's inner-wall, as well as tube-toparticle diameter - for instance by extending the study of Ref. [13] for the system of silo and tube - is required.

The authors gratefully acknowledge the financial support provided by Natural Science Foundation of China (Grant No. 51206113, 51176128 and 51576130) and Shanghai Science and Technology Commission, China (Grant No. 13DZ2260900). We also thank the German Research Foundation (DFG) for funding through the Cluster of Excellence "Engineering of Advanced Materials", ZISC, FPS, the Collaborative Research Center SFB814, and grant PO472/20-2. We gratefully acknowledge the computing time granted by the John von Neumann Institute for Computing (NIC) and provided on the supercomputer JUROPA at Jülich Supercomputing Centre (JSC).

\section{References}

1. H.M. Jaeger, S.R. Nagel, R.P. Behringer, Phys. Today 49, 32-38 (1996)

2. C.P. Liu, P. Wu, L. Wang, Soft Matter. 9, 47624766 (2013)

3. C.P. Liu, F.W. Zhang, P. Wu, L. Wang, Powder Technol. 259, 137-143 (2014)

4. F.W. Zhang, L. Wang, C.P. Liu, P. Wu, Acta Phys. Sin. 63, 014501 (2014)

5. Y. Liu, J.H. Zhao, Chinese Phys. B 24, 034502 (2015)

6. P.A. Cundall, O.D. Strack, Geotechnique 29, 47-65 (1979)

7. C. Kloss, C. Goniva, A. Hager, S. Amberger, S. Pirker, Prog. Comput. Fluid Dy. 12, 140-152 (2012)

8. N.V. Brilliantov, F. Spahn, J.M. Hertzsch, T. Pöschel, Phys. Rev. E 53, 5382-5392 (1996)

9. P. Müller, T. Pöschel, Phys. Rev. E 84, 021302 (2011)

10. C.H. Rycroft, A.V. Orpe, A. Kudrolli, Phys. Rev. E 80, 031035 (2009)

11. J. Ai, J.F. Chen, J.M. Rotter, Powder Technol. 206, 269-282 (2011)

12. F. Fan, E.J.R. Parteli, T. Pöschel, Phys. Rev. Lett. (submitted, 2016).

13. F. Verbücheln, E.J.R. Parteli, T. Pöschel, Soft Matter 11, 4295-4305 (2015)

14. I. Sanchez, O. Pozo, J.R. Darias, Int. Conf. Agricultural Eng. CIGR-AgEng2012, Valencia, 812 July (2012) 